\title{
Late onset of atypical paroxysmal non-kinesigenic dyskinesia with remote history of Graves' disease
}

\author{
Abdul Qayyum Rana, Ambreen Nadeem, Muhammad Saad Yousuf ${ }^{1}$, Zakerabibi M. Kachhvi ${ }^{1}$ \\ Parkinson's Disease and Movement Disorders Center, ${ }^{1}$ University of Toronto, Scarborough Campus, Toronto, Canada
}

\section{ABSTRACT}

Paroxysmal non-kinesigenic dyskinesia (PNKD) is a rare hyperkinetic movement disorder and falls under the category of paroxysmal movement disorders. In this condition, episodes are spontaneous, involuntary, and involve dystonic posturing with choreic and ballistic movements. Attacks last for minutes to hours and rarely occur more than once per day. Attacks are not typically triggered by sudden movement, but may be brought on by alcohol, caffeine, stress, fatigue, or chocolate. We report a patient with multiple atypical features of PNKD. She had a 7-year history of this condition with onset at the age of 59, and a remote history of Graves' disease requiring total thyroidectomy. The frequency of attacks in our case ranged from five to six times a day to a minimum of twice per week, and the duration of episode was short, lasting not more than 2 min. Typically, PNKDs occur at a much younger age and have longer attack durations with low frequency. Administering clonazepam worked to reduce her symptoms, although majority of previous research suggests that pharmacological interventions have poor outcomes.

Key words: Dystonia, Graves' disease, movement disorder

\section{Introduction}

Paroxysmal dyskinesia (PxD) is a group of movement disorders that are characterized by episodes of involuntary movement which may include dystonia, chorea, athetosis, or ballism, without any changes in consciousness. ${ }^{[1]}$ Many classifications for these disorders have been proposed based on various factors. However, the most widely accepted classification based on events that precipitate the attack divide PxD into three main categories: paroxysmal kinesigenic dyskinesia (PKD), paroxysmal non-kinesigenic dyskinesia (PNKD), and paroxysmal exercise-induced dyskinesia (PED). ${ }^{[2-5]}$ Etiologically, PNKD may be a primary disorder and often familial, or it may have a secondary acquired cause which can be sporadic or triggered by alcohol, caffeine, stress, or strong emotions. ${ }^{[6]}$ When diagnosing PNKD, all secondary causes should be considered and ruled out prior to making a diagnosis of primary PNKD.

\begin{tabular}{|l|l|}
\hline \multicolumn{2}{|c|}{ Access this article online } \\
\hline Quick Response Code: & Website: \\
\hline & www.ruralneuropractice.com \\
\cline { 2 - 3 } & \\
\hline & \\
\hline
\end{tabular}

\section{Case Report}

A 66-year-old right-handed female with a history of Graves' disease and total thyroidectomy at the age of 30 was referred to our clinic with the chief complaint of abnormal movements of the left hand. She reported intermittent abnormal posturing and tightening of her left hand. These movements started 6-7 years before she presented to the neurologist. They occurred twice per week and lasted from 1 to $2 \mathrm{~min}$. Furthermore, these movements tended to be unilateral and involuntary, with no change in her level of consciousness during attacks. She reported no aura prior to the episode, while at other times she sensed them coming. She did not specifically recall any triggers that may have preceded the attack, such as sudden movement, substance, or stress, but had experienced that these attacks were much more often than baseline after fatigue or lifting heavy objects. Although she experienced discomfort with these events, she did not experience pain, numbness, weakness, or tingling. She dealt with attacks by spreading her fingers and pushing them against a surface. She had no difficulty performing daily activities, and during episodes, she was able to communicate and was perfectly coherent. There was no family history of movement disorders. Her neurological examination was completely normal.

Address for correspondence:

Dr. Abdul Qayyum Rana, 111-1371 Neilson Rd, Toronto, Toronto, ON M1B 4Z8, Canada. E-mail: ranaaq@yahoo.com 
Furthermore, laboratory investigation including complete blood count, sedimentation rate, renal and liver function tests, thyroid profile, growth hormone, cortisol, and ceruloplasmin levels did not reveal any abnormality. A magnetic resonance imaging (MRI) of brain and a 48-h ambulatory electroencephalogram (EEG) with two clinical episodes were normal.

\section{Discussion}

PxD is a class of heterogeneous motor disorders characterized by abnormal involuntary movement with no alteration to consciousness. It is usually underdiagnosed if not delayed-diagnosed, as the patients are normal between episodes.

Interestingly, there are several atypical features in our case. PNKD usually occurs at a younger age, but in our case, the age of onset was 59 years. The typical duration of attacks is much longer in most patients and may sometimes last up to few hours; however, in our patient, the duration of attacks was only up to $2 \mathrm{~min}$. In typical patients of PNKD, frequency of attacks rarely exceeds more than once per day, whereas in our patient, the frequency of attacks ranged from five to six times a day to a minimum of twice per week.

Although PNKD is an extremely rare movement disorder, very few cases of atypical PNKDs with underlying cerebral structural abnormalities have been mentioned in the literature so far. One case report presented a patient with a 7-year history of atypical PNKD with intracerebral calcifications secondary to hyperthyroidism. ${ }^{[7]}$ Another case report described a patient who had a 6-month history of PNKD with a causative lesion involving subcortical white matter ischemia ${ }^{[8]}$ In another report, a 23-year-old patient with episodes of PNKD was detected to have intracerebral calcifications in multiple brain regions.${ }^{[9]}$ Our case is unique from previous case reports in the literature regarding PNKD, as there are no cerebral structural abnormalities seen in our patient. An MRI of the brain and a 48-h ambulatory EEG with two clinical episodes were normal.

Due to their atypical and unusual clinical presentations, rare paroxysmal movement disorders have frequently been labeled and misdiagnosed as a psychogenic movement disorder. ${ }^{[5]}$ This may also be due to a lack of awareness among physicians. Our patient did not have any psychiatric symptoms. In 1995, Dermirkiran and Jankovic ${ }^{[10]}$ associated a wide range of etiologies to PNKD, for example, autoimmune, vascular, traumatic, infective, and endocrine disorder. Our case, however, was most likely idiopathic because all the investigations including psychiatric symptoms, neuroimaging, and EEG were unremarkable, except that she had a remote history of Graves' disease.

Graves' disease is a known cause of secondary PNKD. Once treated, the symptoms of thyrotoxicosis resolve and the known autoimmune status may improve. However, it is possible that remote history of Graves' disease may cause abnormal autoimmune status not detectable in routine laboratory assays. Although treated successfully, our patient did have a history of Graves' disease requiring total thyroidectomy.

The response of PNKDs to pharmacological treatment is considered poor, but in our case, clonazepam was effective. However, Kaufman and Mink ${ }^{[11]}$ proposed that pallidal deep brain stimulation may be successful with promising results for patients suffering from refractory PNKD. Despite the fact that the age of onset, clinical presentation, and triggering factors of movement disorders are extremely variable, this case clearly highlights the importance of considering the history of Graves' disease in PNKD, even if this is remote.

\section{References}

1. Yamada K, Goto S, Soyama N, Shimoda O, Kudo M, Kuratsu J, et al. Complete suppression of paroxysmal nonkinesigenic dyskinesia by globus pallidus internus pallidal stimulation. Mov Disord 2006;21:576-9.

2. Khan WU, Staios G, Rana AQ. Paroxysmal kinesigenic dyskinesia in a mother and daughter. Acta Neurol Belg 2010;110:201-2.

3. Kailash PB. Paraxysmal dyskinesias. Mov Disord 2011;26:1157-65.

4. Spacey S, Adams P. Familial Paroxysmal Kinesigenic Dyskinesia. In: Pagon RA, Bird TD, Dolan CR, Stephens K, Adam MP, editors. Seattle (WA): GeneReviews Publisher; 1993.

5. van Rootselaar AF, Schade van Westrum S, Velis DN, Tijssen MA. The paroxysmal dyskinesias. Pract Neurol 2009;9:102-9.

6. Abhishek, Anand KS, Premsagar IC. Sporadic paroxysmal nonkinesigenic dyskinesia: A frequently-misdiagnosed movement disorder. Singapore Med J 2007;48:e250-2.

7. Norlinah MI, Shahizon AM. Paraxysmal dyskinesia as an unusual and only presentation of subcortical matter ischaemia: A report of two cases. Med J Malaysia 2008;63:410-2.

8. Alemdar M, Iseri P, Selekler M, Komsuoglu SS. Levetiracetam-respondign paraxysmal nonkinesigenic dyskinesia. Clin Neuropharmcol 2007;30:241-4.

9. Alemdar M, Selek A, İşeri P, Efendi H, Komsuoğlu SS. Fahr's disease presenting with paroxysmal non-kinesigenic dyskinesia: A case report. Parkinsonism Relat Disord 2006;14:69-71.

10. Demirkiran M, Jankovic J. Paroxysmal dyskinesias: Clinical features and classification. Ann Neurol 1995;38:571-9.

11. Kaufman CB, Mink JW, Schwalb JM. Bilateral deep brain stimulation for treatment of medically refractory paroxysmal nonkinesigenic dyskinesia. J Neurosurg 2010;112:847-50.

How to cite this article: Rana AQ, Nadeem A, Yousuf MS, Kachhvi ZM. Late onset of atypical paroxysmal non-kinesigenic dyskinesia with remote history of Graves' disease. J Neurosci Rural Pract 2013;4:449-50.

Source of Support: All work was completely voluntary. Conflict of Interest: None declared. 